\title{
Current and future approaches for the therapeutic targeting of metastasis (Review)
}

\author{
ELITZA GERMANOV ${ }^{1}$, JASON N. BERMAN ${ }^{1,2}$ and DUANE L. GUERNSEY ${ }^{3}$ \\ ${ }^{1}$ Department of Microbiology and Immunology, Faculty of Medicine, Dalhousie University, Sir Charles Tupper \\ Medical Building, 5850 College Street, Halifax, Nova Scotia B3H 1X5; ${ }^{2}$ Department of Pediatrics, Division of \\ Hematology and Oncology, Faculty of Medicine, Dalhousie University, IWK Health Center, PO Box 9700, \\ 5980 University Avenue, Halifax, Nova Scotia B3K 6R8; ${ }^{3}$ Department of Pathology, Division of Molecular \\ Pathology and Molecular Genetics, Faculty of Medicine, Dalhousie University, Sir Charles Tupper \\ Medical Building, 5850 College Street, Halifax, Nova Scotia B3H 1X5, Canada
}

Received July 19, 2006; Accepted August 30, 2006

\begin{abstract}
Metastasis is the process whereby cancer cells disseminate and establish secondary tumors at distant sites from the primary tumor and is estimated to be responsible for $\sim 90 \%$ of all cancer deaths. Cancers with metastatic spread are frequently resistant to conventional chemotherapeutic approaches, underlining the urgent need for novel treatments in these diseases. Recent advances in understanding the mechanisms underlining both the intrinsic cellular and extrinsic micro-environmental factors contributing to the metastatic process have resulted in the identification of a number of molecular targets for the development of specific antimetastatic therapeutic strategies. These targets include intracellular enzymes such as the protein tyrosine kinases, cell surface receptors and their ligands, and elements of the extracellular matrix such as pro-angiogenic factors, protease enzymes and cytokines. Many of these pathways interact with each other, with the possibility of multiple downstream antineoplastic consequences as well as the potential for synergistic effects by targeting more than one of these factors. This review outlines several of the promising targets, and provides examples, of how these targets are being exploited as anti-metastatic therapies in conjunction with conventional treatments.
\end{abstract}

Correspondence to: Elitza Germanov, Department of Microbiology and Immunology, Faculty of Medicine, Dalhousie University, Sir Charles Tupper Medical Building, 5850 College Street, Halifax, Nova Scotia B3H 1X5, Canada

E-mail: germanov@dal.ca

Key words: angiogenesis, chemokines, G protein coupled receptors, glycans, metastasis, matrix-metalloproteinases, neurotransmitters, protein tyrosine kinases, therapeutic targets

\section{Contents}

1. Introduction

2. Tenets of current cancer therapy

3. Intrinsic/cell autonomous targets

4. Extrinsic/environmental factors

5. Conclusions

\section{Introduction}

Cancer is a pathological condition stemming from uncontrolled cell proliferation resulting in the development of a primary tumor, which may then invade into neighboring tissues, and ultimately, spread or metastasize to other parts of the body resulting in secondary metastatic tumor deposits. Carcinogenesis involves a multi-step breakdown or deregulation in normal cellular function brought on by the accumulation of genetic errors in vital cellular regulatory pathways. The behavior of a cell is influenced by a number of factors including: proliferation, differentiation, cell death or apoptosis, cell to cell contacts, association with the extracellular matrix (ECM), immune attack, and the available blood supply (reviewed in ref. 1). Current theory postulates that tumors originate from cells that have acquired a series of abnormalities or mutations involving genes whose protein products govern the cell cycle and its regulatory checkpoint mechanisms, normal cellular differentiation, and inherent proliferative capacity (2). Deficiencies in regulatory cellular processes, such as mitotic-checkpoint regulation, telomere maintenance, and centrosome function, provide increased chances for the accumulation of mutations that further the survival and proliferation of a cancer cell (2). This cell can subsequently resist normal proliferative and survival constraints, such as contact inhibition and anchorage dependence, resulting in a cell with a proliferative advantage over its neighbors. The formation of new blood vessels, angiogenesis, around the primary tumor site is also required in order for a growing tumor to obtain nutrients and oxygen, dispel waste products, and increase in size $(1,3)$. 
Metastasis is the process by which tumor cells migrate from the primary tumor and form secondary tumors at distant sites of the body. Metastasis is a sequential multi-step process requiring the disruption of local cell-cell and cell-ECM interactions, invasion into neighboring tissues, destruction of basement membranes, dissociation and intravasation into blood or lymphatic vessels, survival in the vessel and embolisation to a distant location, extravasation from those vessels into the surrounding tissues, and growth of the metastases accompanied by the generation of new vasculature (Fig. 1) (reviewed in refs. 1,4,5). Metastases can spread via three general routes: local invasion into nearby tissues, hematogenous spread via the blood vessels or lymphatic spread via regional lymph nodes to the rest of the lymphatic system. This review will largely focus on targeting mechanisms of hematogenous spread.

Both cellular intrinsic and extrinsic factors play key roles in tumor initiation, progression and metastasis. Intrinsic factors are cell autonomous and comprise genetic abnormalities activating key oncogenes, or inactivating tumor suppressor genes resulting in deregulation of important pathways involved in cellular growth, proliferation and differentiation. These abnormally regulated cells then interact with extrinsic factors including pro-angiogenic factors and soluble molecules such as chemokines and neurotransmitters. These extrinsic factors exert their influence on the cancer cell by modifying the tumor micro-environment fostering tumor growth, migration, and ultimately metastasis.

\section{Tenets of current cancer therapy}

Surgical excision of the primary tumor in combination with adjuvant treatment using multi-agent chemotherapy and/or radiotherapy is the standard treatment of many cancers. Many chemotherapeutic agents exert their mechanism of action by sabotaging DNA replication and cell division in the rapidly dividing cancer cells, and often rely on the inherent property of a cell to undergo apoptosis when DNA damage is 'sensed' to be of a magnitude that cannot be repaired. However, it is now recognized that resistance to cell death, particularly apoptotic cell death, is a common component of tumorigenesis, metastatic progression, and resistance to chemotherapy (2). Host cellular and tissue responses and the tumor microenvironment may also significantly contribute to chemoresistance (2). Thus, tumor cells may be unaffected by chemotherapy, remaining dormant only to grow into new tumors at a later time. These tumors may subsequently develop metastases advancing the clinical stage of the tumor and making eradication more difficult. Metastatic spread is the most significant poor prognostic factor in the majority of solid tumors. It is estimated that $\sim 90 \%$ of all cancer patients die from metastases underlining the urgent need for treatments preventing the development of metastases (6). As cancer growth, invasion, and metastasis are complex cellular processes, there are numerous strategies for targeting the cancer cells themselves, as well as micro-environmental factors that promote tumor-cell growth, survival, angiogenesis, invasion and dissemination. This review will focus on current therapeutic approaches targeting key events in the metastatic process (Fig. 1) and future strategies that may be employed to prevent the formation of metastases (Table I). This review

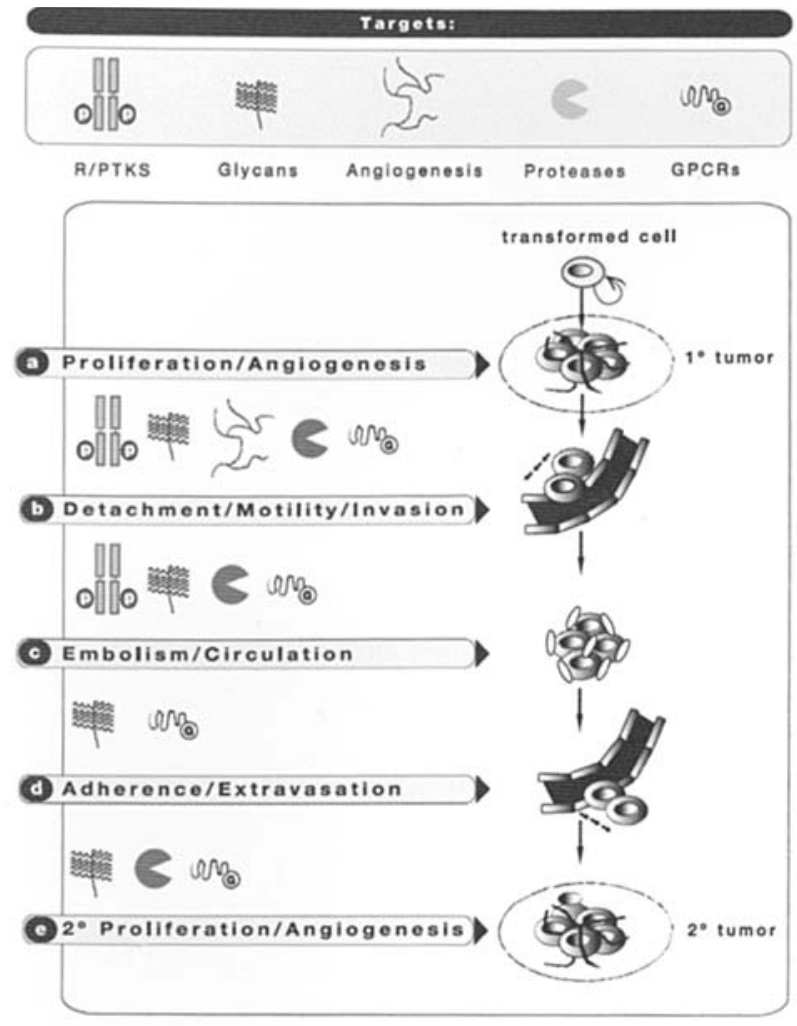

Figure 1. The key stages of tumor progression. Tumor proliferation coupled with angiogenesis is crucial in the early stages of progression (a). Malignant tumor cells detach from the primary $\left(1^{\circ}\right)$ tumor, degrade the extracellular matrix and migrate through the basement membrane into the circulation (b). In the circulation tumor cells form aggregates with platelets and lymphocytes and are transported to secondary $\left(2^{\circ}\right)$ locations (c). Tumor cells adhere to either capillary endothelial cells or to exposed basement membranes and subsequently extravasate into the tissues (d). Proliferation coupled with tumor angiogenesis in the $2^{\circ}$ location completes the metastatic process (e). The therapeutic targets discussed in this review play roles in tumor progression as indicated. Adapted from refs. 1 and 5.

does not aim to be an exhaustive list of current and developing therapies, but will rather highlight how targeting specific pathways in cancer initiation and progression may be effective for the prevention and treatment of metastasis. Ultimately these treatments may replace, or more likely be used in combination with conventional chemotherapy leading to improved outcomes, lower toxicity and a better quality of life for patients with metastatic cancers.

\section{Intrinsic/cell autonomous targets}

Target: protein tyrosine kinases. Members of the receptor or non-receptor protein tyrosine kinase (PTK) family are known to play a crucial role in signal transduction pathways that regulate cell proliferation and differentiation. PTKs and their ligands are often overexpressed in a variety of tumors, and PTK activities are often upregulated or altered leading to abnormal signaling (7). Their deregulation has been linked to carcinogenesis and tumor progression through the constitutive activation of downstream pathways involved in controlling the cell cycle. However, it is increasingly recognized that PTKs also have a key role in mediating angiogenesis, tumor cell dissemination, and cell motility (8). PTKs potently upregulate angiogenic cytokines and matrix-metalloproteinases through 
Table I. Current therapeutic targets of metastasis.

\begin{tabular}{|c|c|c|c|c|}
\hline $\begin{array}{l}\text { Metastatic } \\
\text { target }\end{array}$ & Examples of agents & Mode of action & Examples of cancers targeted & $\begin{array}{l}\text { Related } \\
\text { references }\end{array}$ \\
\hline \multirow[t]{3}{*}{ PTKs } & Trastuzumab & $\begin{array}{l}\text { Monoclonal antibody that blocks } \\
\text { EGFR-ligand interaction resulting } \\
\text { in a signaling blockade }\end{array}$ & $\begin{array}{l}\text { Metastatic breast, oesophageal } \\
\text { and pancreatic carcinomas }\end{array}$ & $(2,25-27)$ \\
\hline & $\begin{array}{l}\text { PKI 166, gefinitib, } \\
\text { erlotinib }\end{array}$ & $\begin{array}{l}\text { Inhibits ATP binding to EGFR } \\
\text { to block signaling }\end{array}$ & $\begin{array}{l}\text { Tumors expressing mutant EGFR } \\
\text { or abberant EGFR signaling }\end{array}$ & $(7,9,14-16)$ \\
\hline & Imatinib mesylate & $\begin{array}{l}\text { Inhibits ATP binding to Abl kinase } \\
\text { and the RTKs of PDGF, SCF } \\
\text { and c-KIT to block signaling }\end{array}$ & CML, GIST & $(9,28-30,32)$ \\
\hline \multirow[t]{5}{*}{ Glycans } & Heparin & $\begin{array}{l}\text { Inhibits heparanase; inhibits growth } \\
\text { factor and heparan sulfate interactions }\end{array}$ & $\begin{array}{l}\text { Metastatic gastrointestinal, } \\
\text { ovarian and breast cancers }\end{array}$ & $(5,110)$ \\
\hline & PI88 & Inhibits heparanase and FGF release & Melanoma, multiple myeloma & $(5,40)$ \\
\hline & Theratope & $\begin{array}{l}\text { Targets tumor glycans, enhances both } \\
\text { humoral and cell-mediated immune } \\
\text { responses }\end{array}$ & $\begin{array}{l}\text { Metastatic breast, ovarian } \\
\text { and colorectal cancer }\end{array}$ & $(5,42-44)$ \\
\hline & $\begin{array}{l}\text { Conjugated } \\
\text { vaccines against } \\
\mathrm{G}_{\mathrm{D} 2} \text { or } \mathrm{G}_{\mathrm{M} 2}\end{array}$ & & $\begin{array}{l}\text { Melanomas, neuroblastomas, } \\
\text { and breast carcinomas }\end{array}$ & $(5,40,45)$ \\
\hline & $\begin{array}{l}\text { Dendritic cells } \\
\text { loaded with } \\
\alpha \text {-GalCer }\end{array}$ & NKT cell activation & $\begin{array}{l}\text { NSCLC cancer or recurrent lung } \\
\text { cancers }\end{array}$ & $(47-51,111)$ \\
\hline \multirow[t]{4}{*}{ Angiogenesis } & Bevacizumab & $\begin{array}{l}\text { Monoclonal antibody that blocks } \\
\text { receptor-VEGF interaction resulting } \\
\text { in a signaling blockade }\end{array}$ & $\begin{array}{l}\text { Metastatic colorectal and renal } \\
\text { cancer, NSCLC }\end{array}$ & $(59-65)$ \\
\hline & PTK 787 & Inhibits VEGF RTK signaling & $\begin{array}{l}\text { CML, acute myelogenous leukemia, } \\
\text { metastatic colorectal cancer }\end{array}$ & $(9,14,66)$ \\
\hline & $\begin{array}{l}\text { Celecoxib, COX } 2 \\
\text { inhibitors }\end{array}$ & $\begin{array}{l}\text { Downregulate the expression of VEGF } \\
\text { and bFGF }\end{array}$ & $\begin{array}{l}\text { Colon, lung, oesophageal, } \\
\text { pancreatic,liver, breast, prostate, } \\
\text { and cervical adenocarcinomas }\end{array}$ & $(53,67,75-77)$ \\
\hline & $\begin{array}{l}\text { Angiostatin } \\
\text { Endostatin }\end{array}$ & $\begin{array}{l}\text { Inhibits angiogenesis, endothelial } \\
\text { cell migration and proliferation, } \\
\text { inducesendothelial cell apoptosis } \\
\text { and cell cycle arrest }\end{array}$ & NSCLC and melanoma & $(79-84)$ \\
\hline \multirow[t]{4}{*}{ GPCRs } & AMD3100 & Anti-CXCR4 antibody & Multiple myeloma & $(95,101)$ \\
\hline & $\begin{array}{l}\text { CXCR4 } \\
\text { antagonist }\end{array}$ & $\begin{array}{l}\text { Antibody that blocks receptor-ligand } \\
\text { interaction resulting in a signaling } \\
\text { blockade }\end{array}$ & & $(37,100)$ \\
\hline & ß blockers & Neurotransmitter antagonists & $\begin{array}{l}\text { Pre-clinical (currently used for the } \\
\text { treatment of cardiovascular diseases) }\end{array}$ & $(107)$ \\
\hline & GABA & $\begin{array}{l}\text { Inhibits increase in cyclic AMP, } \\
\text { inhibiting the promigratory effect } \\
\text { of norepinephrine }\end{array}$ & Pre-clinical & $(108,109)$ \\
\hline
\end{tabular}

the mitogen-activated protein kinase (MAPK) and phosphoinositide 3-kinase (PI3K) pathways which both contribute to the development of metastases (9). A number of drugs have been designed to target PTKs by a variety of mechanisms including the inhibition of receptor-ligand interactions and the blockade of PTK signaling by inhibiting ATP binding (7). 
Epidermal growth factor receptor and other members of $\operatorname{ErbB}$ (HER) family. The epidermal growth factor receptor (EGFR), also known as ErbB(1) or HER1, a member of the human ErbB receptor family of receptor tyrosine kinases (RTKs), is expressed in a wide range of solid tumors, and is frequently associated with a poor prognosis $(8,9)$. The ErbB family influences cancer progression through at least three major mechanisms: overexpression of ErbB ligands, amplification of $\operatorname{ErbB}$ genes, and mutational activation of $\operatorname{ErbB}$ genes (10). Overexpression and/or aberrant signaling of the ErbB family of RTKs affects a number of cellular processes via the $R A S$ oncogene pathway including: cellular proliferation; activation of the protein kinase $\mathrm{B}$ (PKB) pathway, which in turn leads to cell cycle progression via activation of Cyclin D1; upregulation of specific matrix-metalloproteinases (MMPs), inducing breakdown of the extracellular matrix thus promoting tumor spread; and prevention of apoptosis by inhibiting the actions of the pro-apoptotic proteins, FAS ligand (FASL) and BAD $(8,9)$. The EGFR ligand, EGF, is also linked to tumor angiogenesis, as it functions as a strong inducer of the proangiogenic vascular endothelial growth factor (VEGF) (11). Indeed, the overexpression of EGF or EGFR by human pancreatic tumors and breast tumors correlates with rapid progression and metastatic disease (12). Furthermore, expression of EGFR appears to provide protection to malignant tumor cells from the cytotoxic effects of chemotherapy and radiotherapy, making these treatments less effective $(7,13)$. Inhibition of EGFR and/or other members of the ErbB family or their respective ligands is an appealing target for anticancer/ antimetastatic therapy.

PKI 166 is a dual inhibitor of both EGFR (ErbB1) and ErbB2 (HER2). This drug interacts with the intracellular domain of the ErbB receptors by competitively inhibiting ATP binding, which is necessary for effective signaling $(9,14)$. Early experiments showed that oral administration of PKI 166 three times a week, in combination with gemcitabine chemotherapy, inhibited the growth and metastasis of orthotopically implanted human pancreatic carcinoma, in nude (athymic) mice (14). Immunohistochemical analysis of pancreatic tumors from mice treated with PKI 166 revealed that the decrease in activated EGFR was accompanied by a decrease in expression of proangiogenic molecules VEGF and interleukin-8 (IL-8) (14). In addition, in another rodent model, Kim et al (15) showed that the combined administration of PKI 166 and the drug paclitaxel to nude mice with orthotopically implanted human prostate cancer cells blocked EGFR signaling in the tumor and neighboring endothelial cells, and resulted in reduced incidence and size of metastatic bone lesions, a common metastatic site in this type of cancer. The combination treatment also significantly induced apoptosis of EGFR expressing endothelial cells within tumors indicating anti-angiogenic implications (15). PKI 166 has been tested in early phase clinical trials in patients with a wide variety of tumors identified as having EGFR overexpression including carcinoma of the breast, head and neck, prostate, urinary bladder, GI tract, and gliomas (16) (reviewed in ref. 9).

Iressa ${ }^{\circledR}$ (gefitinib) and Tarceva ${ }^{\circledR}$ (erlotinib) are drugs currently approved for the treatment of patients with advanced or metastatic non-small cell lung cancer (NSCLC), breast cancer, and head and neck cancers and function by targeting the EGFR pathway. These drugs act in a similar manner to PKI 166 by competing for ATP binding to the kinase domain, and thus directly blocking intracellular growth signals (7). These drugs inhibit aberrant signaling due to mutations in the EGFR gene, and the resulting downstream signaling events, such as activation of the PKB/AKT survival responses and RAS-signaling pathways $(10,13)$. Clinical trials with gefitinib for advanced NSCLC have shown improved disease stabilization in $>42 \%$ of patients so that almost half of the patients treated with gefinitib in both trials benefited from treatment $(7,17,18)$. Erlotinib has similarly provided beneficial treatment to patients with advanced chemotherapy-resistant NSCLC (7). Curiously, the response to gefinitib and erlotinib does not directly correlate with tumor EGFR expression levels (13). However, it is likely that a positive response to gefinitib and/or erlotinib is related to EGFR activating mutations or genetic alterations, EGFR genetic copy number, or the level of EGFR PTK activity $(10,13,19-21)$. EGFR mutations were found in 8 out of 9 patients with gefinitib-responsive NSCLC, compared to none out of 7 patients with gefinitib-unresponsive NSCLC (19). However, some patients that had shown initial responses to gefinitib or erlotinib later developed resistance to these drugs. Pao et al (22) attributed this resistance to an emergence of tumor cells containing additional EGFR mutations in the presence of gefinitib, suggesting that gefinitibresistance genes may influence patient outcome. In addition, since gefinitib may mediate its actions through the inhibition of EGFR-PTK downstream pathways, such as the PKB signaling pathways, patients whose tumors were positive for phosphorylated PKB (p-PKB) had a better response rate, and slower disease progression when treated with gefinitib than patients whose tumors were negative for p-PKB $(10,23)$. Similarly, mutations in the downstream RAS-signaling genes may influence clinical response to these agents (10). In support of this theory, mutations in K-RAS were associated with a lack of sensitivity to gefinitib or erlotinib in a screen of lung adenocarcinomas (24).

Herceptin ${ }^{\circledR}$ (trastuzumab) is a drug that specifically targets aberrant signaling through the ErbB2 receptor of the ErbB family (2). Trastuzumab is a humanized monoclonal antibody that acts by selectively binding the extracellular domain of the ErbB2 receptor. This binding slows tumor growth and progression by inhibiting growth factor signaling. Trastuzumab is used in combination with paclitaxel chemotherapy to treat patients with ErbB2 positive metastatic breast cancer. The Phase III clinical trials for the treatment of metastatic breast cancer conclusively showed that trastuzumab in combination with standard chemotherapy slows disease progression providing patients with a longer median survival time compared to chemotherapy alone (25). Trastuzumab has since been approved for the treatment of metastatic breast cancer. Trastuzumab is also undergoing clinical trials for the treatment of metastatic esophageal and pancreatic carcinomas that overexpress ErbB2 $(26,27)$.

$B C R-A B L$ tyrosine kinase. Gleevec ${ }^{\circledR}$ (imatinib mesylate) was originally developed for the treatment of Philadelphia chromosome-positive chronic myeloid leukemia (CML). CML is a hematologic stem cell disorder characterized by excessive myeloid proliferation. The Philadelphia chromosome is generated due to a reciprocal translocation between 
chromosome 9 and 22, creating a novel hybrid gene, which produces a BCR-ABL fusion protein with constitutive tyrosine kinase activity (9). Constitutive BCR-ABL tyrosine kinase activity affects numerous signal transduction pathways that are essential for leukemic transformations. Imatinib mesylate acts by blocking the binding site for ATP in the ABL kinase, thereby preventing phosphorylation of tyrosine residues on substrate proteins. Inhibition of phosphorylation prevents the activation of downstream signal transduction pathways that induce the leukemic transformation to CML including RAS, PI3K, and JAK-STAT pathways (28). The Phase III clinical trials confirmed that imatinib mesylate provides significantly improved progression-free survival over a 12-month period compared to the standard treatment, (interferon- $\alpha$ and cytarabine) in chronic phase CML and has truly revolutionized the treatment of this disease (29). Imatinib mesylate is now the standard of care for adult patients with chronic phase CML. The drug is not entirely selective for the BCR-ABL kinase and has also been found to inhibit the RTKs for plateletderived growth factor (PDGF), stem cell factor (SCF) and activated KIT kinase (c-KIT) $(9,30)$. Gastrointestinal stromal tumors (GIST) are rare frequently metastatic tumors that commonly express mutated c-kit or PDGF tyrosine kinases. Imatinib mesylate yielded promising results in patients with these tumors and consequently the drug has been approved for the treatment of GIST in many countries (32). Unfortunately, as is the case for gefinitib, patients may develop disease resistant to imatinib mesylate. This has prompted the development of second generation BCR-ABL tyrosine kinase inhibitors, such as Dasatinib ${ }^{\circledR}$, which has just been approved by the FDA for treatment of CML resistant to imatinib mesylate (31).

Mounting evidence continues to demonstrate the efficiency of PTK inhibitors in a wide range of malignancies. The combination of targeting a protein tyrosine kinase with current chemotherapy approaches appears to be a promising strategy, which is continuing to be developed and optimized.

Target: glycans. Glycans exist as membrane-bound glycoconjugated molecules on the surface of tumor cells or as secreted molecules, which can become integral parts of the ECM. These molecules have been recognized as mediating key pathophysiological events during the various steps of tumor progression (5). The physiological locations of glycans place them in a position to mediate cell adhesion and motility in addition to intracellular signaling events (5). Membranebound glycans are able to bind the lectin family of proteins, which are important in cell-cell recognition, cell-adhesion and motility, and pathogen-host recognition. Many lectins are found on the surface of immune cells, and endothelial cells, and in the ECM as soluble adhesion molecules (5). Through changes in glycosylation, membrane-bound glycans present on the surface of tumor cells can facilitate the detachment of invasive cells from the primary tumor by reducing tumor cellcell adhesion and modifying tumor cell-ECM interactions, $(33,34)$. Similarly, changes in glycosylation and glycan-lectin interactions can facilitate the dissemination of tumor cells through the blood stream by enhancing their adhesion to platelets and immune cells (embolisation), as well as to endothelial cells (5). One well-known glycan family, the gangliosides, including $\mathrm{G}_{\mathrm{D} 3}$ and $\mathrm{G}_{\mathrm{M} 3}$ are commonly overexpressed in melanomas, lung cancers and neurogenic tumors (33) and can enhance metastatic progression through their ability to regulate growth signaling through interactions with RTKs (5). Another group, the glycosaminoglycans, can also function as co-receptors for pro-angiogenic molecules and tumor growth factors, such as bFGF, VEGF, TGFß, and IL-8, facilitating tumor growth and angiogenesis $(5,35)$. Furthermore, a particular glycan family, the heparan-sulphate proteoglycans (HSPGs), have the ability to bind several chemokines and assist the establishment of chemokine gradients across vascular endothelial layers, thus facilitating extravasation and intravasation of tumor cells out of or into blood or lymphatic vessels as is the case with some breast cancer cells $(36,37)$. In addition, the degradation of the heparansulphate chains of certain HSPGs by heparanases released by migrating tumor cells can aid tumor progression by liberating sequestered growth factors (38). This process of heparanase release correlates with metastatic potential in a number of tumors prompting the development of strategies aimed at inhibiting heparanase activities $(5,39)$.

The anticoagulant drug, heparin, can effectively inhibit heparanase and interactions between growth factors and heparan sulfates; however, its use can lead to significant adverse side effects, particularly hemorrhage (5). Another heparanase inhibitor, PI88, is currently in Phase II clinical trials in melanoma and multiple myeloma (40). Roy et al (41) showed that adenoviral vectors containing antisense sequences to the heparanase gene could effectively silence heparanase actions in human malignant $70 \mathrm{~W}$ and murine B16 B15b melanoma cells. Correlative in vivo studies demonstrated that a heparanase gene knockdown resulted in the absence of human malignant $70 \mathrm{~W}$ melanoma pulmonary metastases in nude mice (41).

Tumor glycans can also be exploited as targets for eliciting anti-tumor immunity against metastatic tumors. One example is the development of antibodies that augment or boost the immune response, by targeting tumor surface mucin glycans (e.g. MUC1 or MUC16) on tumor cells, thereby inhibiting tumor proliferation and invasion (5). Glycans themselves are poor immunogens, but the aberrant clustering of glycosylated mucins on the tumor cell surface provides sites for the attachment of antibodies, thus improving their activity as immunogenic agents. Additionally, vaccines are being developed that incorporate glycans that are specifically overexpressed on the surface of tumor cells. These vaccines are constructed by conjugating a particular glycan to a carrier protein, such as keyhole limpet haemocyanin (KLH) (5). The vaccines are often administered in combination with an adjuvant that will enhance $\mathrm{T}$ cell responses directed towards the glycan moiety (5). Theratope ${ }^{\circledR}$ is one such vaccine, consisting of conjugated sialyl-Tn mucin tumor antigens to KLH, which elicits both humoral (B cell) and cell-mediated (T cell) immune responses (5). A Phase III clinical trial for the treatment of advanced metastatic breast cancer with Theratope following chemotherapy showed a positive survival effect over placebo in a subset of patients (42). In addition, this vaccine is currently being examined in the treatment of patients with ovarian and colorectal cancer $(43,44)$. Similarly, conjugated vaccines have been developed against the 
glycosphingolipids, $\mathrm{G}_{\mathrm{D} 2}$ and $\mathrm{G}_{\mathrm{M} 2}$, that are characteristically overexpressed in several cancer types, such as melanomas, neuroblastomas, and breast carcinomas, and are currently being tested in ongoing clinical trials $(5,40,45)$.

The marine sponge derived glycosphingolipid, $\alpha$-galactosyl ceramide ( $\alpha$-GalCer), is very antigenic and specifically activates a specialized lymphocyte population termed natural killer T (NKT) cells (46). NKT cells are activated when $\alpha-\mathrm{GalCer}$ is presented to NKT cells by antigen presenting cells in the context of the antigen presenting molecule CD1d (46). NKT cell activation results in the sequential activation of effector cells such as natural killer (NK) cells and cytotoxic T lymphocytes (CTLs), which have known antitumor activities (reviewed in ref. 47). A number of studies in murine models showed that NKT cell activation by $\alpha-G a l C e r$ has antimetastatic properties, inhibiting experimental metastatic spread to the liver and lungs for various tumor types, including melanoma and lung carcinoma (48-50). A Phase I clinical trial for the treatment of patients with NSCLC cancer or recurrent lung cancers utilized antigen presenting dendritic cells which have their CD1d surface molecules coated with $\alpha$-GalCer and demonstrated disease stabilization, minimal side effects and a good quality of life for greater than one year in several patients (51).

The fact that many tumor cell types overexpress surface glycans such as chondroitin-sulphate proteoglycans (CSPGs) and glycosphingolipids has led to research aimed at the development of tumor-specific drug delivery. Targeting treatments to specific pathological sites will reduce systemic toxicity in patients, and likely provide greater drug delivery and efficacy. A study by Lee et al (52) used chondroitin sulphate-binding cationic liposomes containing the chemotherapeutic agent, cisplatin, to suppress both local growth and the development of liver metastasis in mice. This specific targeting of cisplatin was superior in reducing tumor growth, metastasis, drug toxicity, and improving overall survival when compared to the actions of cisplatin administered alone (52). In a similar manner, it is also possible to couple carrier cationic liposomes to monoclonal antibodies against glycosphingolipids (e.g. $\mathrm{G}_{\mathrm{D} 2}$ ) to specifically target drug delivery (5).

Due to the broad spectrum of glycans affecting many stages of tumor proliferation and progression, glycan-specific targeting approaches provide attractive therapeutic strategies, which could be used in combination with standard treatments. Since a specific glycan might be expressed at different stages of tumor progression, glycan-specific targeting approaches might have broad effects on a number of glycan-protein interactions, influencing metastatic spread (Fig. 1).

\section{Extrinsic/environmental factors}

Target: angiogenesis. Angiogenesis is a complex and necessary process for tumor growth, invasion, progression and metastasis (Fig. 1) (reviewed in ref. 53). Like normal tissue, tumors require an adequate supply of oxygen, metabolites, and an efficient way to remove waste products (3). However, these requirements can vary between tumor types and during the course of malignant progression (54). Vasculature within and surrounding tumors also provides a means of transport for tumor cells by allowing them to intravasate and travel to distant sites, in order to establish metastatic deposits (reviewed in ref. 53). Pro-angiogenic endothelial cell gene expression is upregulated by physiological stimuli, such as hypoxia in expanding tumors, and also by oncogene activation or TSG mutation (reviewed in ref. 55). Tumors themselves can express various pro-angiogenic factors aiding their own growth and progression (53). The major pro-angiogenic factors include VEGF, IL-8, EGF, PDGF, basic fibroblast growth factor (bFGF).

Vascular endothelial growth factor. VEGF, a mitogen specific for vascular endothelial cells, is one of the principle pro-angiogenic factors, produced by many cell types, including hematopoietic stem cells, mononuclear cells and secreted by tumor cells and tumor-associated stromal cells $(53,56,57)$. By binding to specific VEGF surface receptors with tyrosine kinase activities, VEGF can induce vascular endothelial cell proliferation, migration, and differentiation leading to neovascular growth $(56,58)$. Inhibition of VEGF-induced angiogenic signals in tumor-associated vessels may potentially halt tumor growth, and prevent metastasis. However, inhibition of angiogenesis does not occur rapidly when compared with the response typical of cytotoxic therapies. The inhibition of angiogenesis may not actually kill tumor cells, but rather slow their progression and provide a means for disease stabilization in otherwise untreatable diseases.

Avastin $^{\circledR}$ (bevacizumab) is a drug that targets the interaction between VEGF and its receptors (VEGFR1 and VEGFR2). Bevacizumab is a humanized monoclonal antibody that binds VEGF, preventing ligand-receptor interactions, and VEGF induced angiogenesis. Bevacizumab has been approved for use in combination with 5-fluorouracil chemotherapy as a first-line treatment in patients with metastatic colorectal cancer (59). The results of a Phase III clinical trial demonstrated that this combination extends survival of patients by nearly 5 months with a median 10.6 months progression-free survival, compared to a median 6.4 months progression-free survival (60). Furthermore, bevacizumab has been tested in clinical trials for the treatment of metastatic renal cell carcinoma with encouraging results demonstrating slower disease progression when compared to placebo $(61,62)$. Combination therapy with bevacizumab and erlotinib has also been shown to be efficacious in renal carcinoma and NSCLC (62-64). A Phase II clinical trial for the treatment of NSCLC tested bevacizumab combined with carboplatin and paclitaxel chemotherapy compared to chemotherapy alone and demonstrated that the addition of bevacizumab improved the overall response rate (65).

PTK787 is a tyrosine kinase inhibitor specifically targeting VEGFR tyrosine kinase signaling of the VEGFRs (reviewed in ref. 9). Pre-clinical data show that PTK787 selectively inhibits VEGF-mediated endothelial cell proliferation, cell survival, and cell migration in vitro (9). In rodent models, PTK787 selectively inhibits VEGF-mediated angiogenesis, and the development of lung and lymph node metastasis $(9,66)$. Baker et al (14) showed that daily oral administration of PTK 787 combined with gemcitabine chemotherapy could inhibit the growth and rate of metastasis of human pancreatic carcinoma cells implanted orthotopically in nude mice (14). A combination of PTK 787 and EGFR kinase inhibitor PKI 
166 together with gemcitabine significantly retarded tumor growth and metastases by inducing apoptosis in both pancreatic tumor cells and tumor-associated vascular endothelial cells, leading to a significant increase of overall survival time (14). Clinical trials with PTK 787 are currently underway for the treatment of metastatic colorectal cancer, CML and acute myelogenous leukemia.

Cyclo-oxygenase 2. Another pathway involved in the induction of angiogenesis is the cyclo-oxygenase 2 (COX2) pathway, which is responsible for prostaglandin $\mathrm{G} / \mathrm{H}$ synthesis via arachidonic acid metabolism (53). Enhanced COX2induced synthesis of prostaglandins stimulates cancer cell proliferation, inhibits apoptosis, and increases metastatic potential (67-71). COX2 is overexpressed in mucosal colon and gastric adenocarcinomas, and is associated with advanced spread to lymph nodes (72-74). Elevated COX2 mRNA and protein levels are similarly associated with advanced stages of esophageal, head and neck, breast, lung and prostate cancers, indicating an intimate involvement of COX2 in tumor progression and metastasis (72).

COX2 inhibitors are anti-inflammatory agents with antiangiogenic activities that downregulate the expression of the pro-angiogenic factors VEGF and bFGF (75). COX2 inhibitors are currently being evaluated in conjunction with chemotherapy and radiotherapy in clinical trials for the treatment of colon, lung, esophageal, pancreatic, liver, breast and cervical cancers (reviewed in ref. 67). Furthermore, COX2 inhibitors have also been considered for the treatment of prostate and cervical adenocarcinomas, which both express high levels of COX2 (53). COX2 inhibitors may be most effective as a single agent in pre-malignant conditions. For example, the COX2 inhibitor Celebrex $^{\circledR}$ (celecoxib) was able to reduce the number of colorectal polyps by $28 \%$ in patients with familial adenomatous polyposis, a syndrome that predisposes patients to colon cancer (76). In combination with paclitaxel, a known inducer of COX2, and carboplatin, a chemotherapy agent, celecoxib was evaluated for the treatment of NSCLC, as a means of enhancing the patient response to chemotherapy. Results of this Phase II clinical trial demonstrated an enhanced overall response rate to paclitaxel and carboplatin with the coadministration of celecoxib (77).

Matricryptins and matrikines. Matrix-metalloproteinases and other proteases generate proteolytic and cryptic fragments from the ECM, which have anti-angiogenic activities (reviewed in ref. 78). Research into these proteolytic and cryptic fragments, termed matrikines and matricryptins, has stimulated interest for their development as anti-angiogenic therapies. The examples listed below are all currently in Phase II clinical trials.

Angiostatin, a plasminogen fragment, is an angiogenic inhibitor that hinders endothelial cell migration and proliferation, and increases endothelial cell apoptosis $(79,80)$. Angiostatin has been reported to suppress metastasis in Lewis lung carcinoma and to induce and sustain dormancy of human and murine primary fibrosarcomas and their metastases in mice $(81,82)$. Endostatin, a fragment of basement membrane collagen (XVIII), inhibits endothelial cell proliferation, migration, angiogenesis and tumor growth, and induces endothelial cell apoptosis and cell cycle arrest (reviewed in ref. 78). Sonveaux et al (83) showed that irradiation, in a dose-dependent manner, might paradoxically induce the activation of pro-angiogenic pathways in endothelial cells. Thus, combining anti-angiogenic treatments such as angiostatin or endostatin with radiotherapy may increase the therapeutic efficacy of radiotherapy (84).

A primary benefit of anti-angiogenic agents is their ease of administration. Most are given orally, are generally well tolerated and have minimal side effects $(64,66,77)$. Additionally, anti-angiogenic agents can be used not only for stunting primary cancer growth, but also for the prevention of cancer recurrence, and metastasis. Combinations of antiangiogenic agents may provide synergistic effects compared to single agents. Due to their slow rate of action, antiangiogenic agents currently do not have a place as first line therapy in aggressive tumors. However, future regimens combining cytotoxic approaches with inhibition of vascular growth may become an important upfront treatment in a number of malignancies.

Target: extracellular matrix. Matrix-metalloproteinases (MMPs) and other proteases, such as heparanase, produced by cancer cells break down the surrounding extracellular matrix (ECM), thereby facilitating cancer cell migration, in addition to promoting tumor angiogenesis, as described above. The ECM is a reservoir of growth factors, which are released by matrix protease-mediated ECM degradation and can further contribute to the growth and progression of a tumor. Several studies have shown that the upregulation of MMPs, and members of the plasminogen activating pathway, such as urokinase plasminogen activator (uPA) and its receptor (uPAR), are involved in the progression of transformed cells into invasive cells and the development of metastases $(78,85)$. These enzymes are required by the growing tumor to degrade the ECM and break through the underlying basement membrane in order to intravasate into surrounding blood or lymphatic vessels. A number of strategies have been directed at preventing the actions of matrix-degrading proteases in an attempt to stop the initial events leading to metastases. Unfortunately, the promising pre-clinical profile of MMPtargeted therapy was not reflective of the results in the clinical setting. The results of several large scale Phase III studies with MMP inhibitors, such as marimastat and prinomastat were discouraging. A Phase III clinical trial for marimastat showed no significant improvement over placebo in progression-free survival or overall survival in the treatment of patients with metastatic breast cancer (86). Similarly, a Phase III clinical trial for prinomastat showed no significant improvements over placebo in time to progression or overall survival in patients with NSCLC leading to early closure of the study (87). However, these clinical trials involved patients with advancedstage disease and these agents may yet have a role in treating cancers that are less advanced.

New alternative strategies to the original small molecule inhibitors that failed are being explored to target matrixdegrading proteases. In a recent study, Rao et al (85) engineered a replication-deficient adenovirus expressing antisense UPAR and antisense MMP-9 transcripts, referred to as Ad-uPAR-MMP-9, and transduced these into H1299 lung cancer cells in an attempt to downregulate UPAR and MMP-9 expression (85). The authors demonstrated inhibition of UPAR 
and MMP-9 expression and decreased invasive capacity in vitro, in addition to inhibition of a capillary-like structure formation by endothelial cells co-cultured with H1299 cells. AD-uPAR-MMP9 also inhibited tumor growth in vivo by shrinking subcutaneous H1299 lung cell tumors in mice. Furthermore, AD-uPAR-MMP9 significantly reduced the metastatic capacity of lung cancer cells in vivo. Another study, also aimed at inhibiting the action of UPAR expression, reported that UPAR is required for invasion and metastasis of highly malignant oral cancer cells. Nozaki et al (88) found that by utilizing antisense oligodeoxynucleotides and small interfering RNAs (siRNAs) targeting uPAR they could inhibit progression of oral cancer cells in a chick embryo assay model of metastasis (88).

Many MMPs and proteases are $\mathrm{pH}$ sensitive. A low extracellular $\mathrm{pH}$ is thought to result in increased secretion and activation of proteases (89). Certain tumor cells have the capability of lowering the $\mathrm{pH}$ in the surrounding milieu by pumping out protons from their cytosol via the V-ATPase proton pump. Expression of V-ATPase on the plasma membrane of some tumor cells correlates with their metastatic potential (90). By acidifying their extracellular environments, tumors upregulate the activity of proteases, which degrade the ECM aiding the tumor cells in invasion and metastasis. A recent study by Lu et al (91), utilizing siRNA to target the ATP6L subunit of the proton pump V-ATPase, showed promising results in suppressing cancer metastasis and hindering tumor growth in a human hepatocellular carcinoma xenograph model in mice. The siRNA targeted knock-down of ATP6L resulted in a downregulation of MMP-2 expression, with decreased tumor invasion and metastasis. MMP-2 has also been implicated as a direct angiogenesis-promoting factor, suggesting that the targeted knock-down of ATP6L also had anti-angiogenic consequences, which likely contributed to the decrease in tumor progression (94). Furthermore, the resulting increase in extracellular $\mathrm{pH}$, caused by the reduction of proton extrusion, is linked to the reduction of some acid-induced angiogenesis-promoting factors such as IL-8 and VEGF $(92,93)$.

Target: G-protein coupled receptors (GPCR) and their ligands. Entschladen et al (6) suggest that the manifestation of a metastatic phenotype, which may have been established in early tumorigenesis by genetic alterations, is regulated by guidance of external signal molecules, such as chemokines or neurotransmitters. These signal molecules may also dictate the final destinations within the body where tumor cells will migrate and form metastatic tumors (6). Active migration of tumor cells is a pre-requisite for tumor cell invasion and metastasis. As a result, much research has been devoted to elucidating the molecular mechanisms that mediate and regulate the migratory activity of tumor cells. The most prominent regulatory factors are ligands to a group of seventransmembrane G-protein coupled receptors (GPCRs), such as chemokines and neurotransmitters (6). By understanding how these receptors regulate migration, it may be possible to block these activities by utilizing agonists to therapeutically modulate and inhibit tumor cell migration, thus cancer invasion and metastasis.
The GPCRs regulate tumor cell migration in a manner analogous to the recruitment and homing of leukocytes. The ligands for these receptors, chemokines or neurotransmitters can induce directed or chemotactic migration of cells bearing the respective receptors. Some tumor cells can initiate GPCR expression by gene mutation, gene fusion, or local conditions such as hypoxia (95). Receptor-ligand interactions induce a number of downstream signaling pathways including, phospholipase $\mathrm{C} B$ isoforms, $\mathrm{PI} 3 \mathrm{~K}$, various Src family kinases, and the small GTPase Rho (reviewed in ref. 96). These signal transduction pathways play crucial roles in the formation of cell polarity, directional chemokine gradient sensing, F-actin polymerization, and myosin assembly, which are all necessary for the directional migration of cells (96). Chemokine receptor-ligand interactions may also induce detachment and dissemination of metastatic cells from the primary tumor, in addition to directing the migration of these cells in an organspecific way. Furthermore, chemokines and their receptors may also be directly involved in the transformation, survival, and growth of tumor cells by delivering anti-apoptotic and proliferative signals $(95,96)$. In addition, there is evidence for interaction with pro-angiogenic stimuli, such as VEGF and hypoxia, which can upregulate the expression of certain chemokines such as $\mathrm{ELR}^{+} \mathrm{CXC}$ chemokines, and their receptors such as CXCR4. Furthermore, these chemokines are potent angiogenic inducers (8). Inflammation has also been implicated in tumor development, growth, progression and metastasis, and may mediate these effects through the release of chemokines from various inflammatory cells present in the tumor micro-environment (97).

Chemokines and their receptors. A number of human and murine cancer cells express the chemokine receptor CXCR4, and it is the most commonly overexpressed chemokine receptor found on cancer cells $(95,98)$. The chemokine CXCL12 is expressed in a wide variety of tissues and its interaction with CXCR4 regulates other functions such as, tumor cell growth and survival, cytokine secretion, cytoskeletal re-arrangement, and anti-apoptotic signals $(95,96,99)$. Two studies have reported increased expression levels of the CXCR4 ligand, chemokine CXCL12, in metastatic tumor foci compared with primary tumors (reviewed in ref. 96). The involvement of CXCR4 in metastasis has been documented for a variety of tumors, including mammary carcinoma $(95,96)$.

Muller et al (37) showed that CXCR4 is highly expressed in mammary carcinoma tissue while its ligand CXCL12 is expressed in other tissues such as the lymph nodes, bone marrow and lungs, suggesting a mode of targeted organspecific metastasis and dissemination of tumor cells. They demonstrated that a neutralizing antibody directed to the CXCR4 receptor could inhibit intravenous or orthotopically induced lung or lymph node metastases in nude mice (37). Similarly, a study by Guleng et al (100) reported that blocking the CXCR4 receptor with a neutralizing antibody resulted in decreased growth of subcutaneously injected gastrointestinal tumors through the suppression of tumor angiogenesis in a murine model (100). This effect was independent of the CXCR4 status of the tumor cells. Anti-angiogenic effects were also suggested by the absence of increased VEGF levels and the determination that endothelial cells in proximity to tumor tissues express CXCR4. Therapy directed toward 
CXCR4, perhaps in combination with anti-angiogenic therapies, may emerge as a promising therapy that may be useful against a broad spectrum of cancers. AMD3100, a CXCR4 antagonist, originally developed for the treatment of human immunodeficiency virus (HIV) infection, is now being considered for the treatment of a number of malignancies (95). In preclinical studies, Rubin et al (101) demonstrated that the systemic administration of AMD3100 inhibits the growth of intracranial glioblastoma and medulloblastoma xenografts in nude mice, by increasing apoptosis and decreasing the proliferation of tumor cells.

The chemokine receptor CCR7, which is the receptor for CCL19 and CCL21 chemokines, also plays an important role in metastasis (reviewed in refs. 95,96). CCR7 expression correlates with metastatic potential and poor prognosis. CCL21 is found in high levels in the regional lymph nodes of patients with a number of malignancies (95). CCR7 expression has been found in breast cancer, gastric carcinoma, NSCLC, esophageal cancers, chronic lymphocytic leukemia, and melanoma (95). Wiley et al (102) established that transducing murine B16 melanoma cells with CCR7 enhanced their metastasis to regional lymph nodes compared to non-transfected B16 melanoma cells. This enhanced metastatic capability was abrogated with a CCL21 neutralizing antibody. In a clinical study, Mashino et al (103) showed that an increase of lymph node metastases and lymph node invasion correlated with expression levels of CCR7 in carcinoma cells in 66\% (42 of 64) of patients with gastric carcinoma. Patients with CCR7-positive tumors had a poorer prognosis compared to those with CCR7-negative tumors. Studies have revealed similar results in patients with esophageal carcinoma (104).

Other chemokine receptors such as CCR3, 4, 5, 10 and CXCR2 play a role in the progression of cancers like melanoma and T-cell leukemia (95). Monoclonal neutralizing antibodies against chemokine receptors have been successfully used for inhibiting the growth and/or spread of malignant tumor cells in pre-clinical models and will hopefully have similar efficacy in the clinical setting $(100,101)$. As with many of these targeted therapies, targeting chemokines is likely to have the greatest benefit when used in combination with therapies targeting other pathways and more standard chemotherapeutic approaches.

Neurotransmitters and their receptors. Neurotransmitters have been known to be involved in tumor-cell proliferation, differentiation, and survival and recent evidence suggests a role in the regulation of tumor-cell migration. Neurotransmitters are thought to function in a manner similar to chemokines (reviewed in ref. 6). Neurotransmitters, such as histamine and bradykinin, have a pro-inflammatory function in addition to pro-migratory activities that may contribute to malignant spread $(105,106)$. Several human tumors including human urinary-bladder carcinoma have been shown to contain nerve fibers suggesting that local delivery of neurotransmitters may contribute in tumor growth and progression (6).

Pharmacological blockade of neurotransmitter release may provide a novel strategy in the prevention of cancer progression and metastatic spread. Targeting neurotransmitters may have several advantages over targeting chemokines. As opposed to chemokines where receptor blockade may impact upon numerous chemokine pathways, due to a number of ligands sharing the same receptor, neurotransmitter receptor blockade can be highly specific $(6,96)$. Moreover, selective antagonists for several neurotransmitters are already available and in broad clinical use for the treatment of other diseases, such as $\beta$ blockers in cardiovascular diseases (6).

An epidemiological study by Andersson et al (107) hints at the potential benefit for neurotransmitter blockade in anticancer treatment. Men treated for hypertension (with $\beta$ blockers and/or other antihypertensive agents) had a higher overall mortality than those who did not have hypertension, but a slightly lower mortality due to cancer. However, this study did not discriminate between patients treated with $\beta$ blockers from those treated with other hypertension drugs, or between the types of cancer.

In addition to neurotransmitter receptor blockade, increasing levels of particular inhibitory neurotransmitters may provide another method to abrogate effects of the promigratory neurotransmitters. A study by Joseph et al (108) showed that the neurotransmitter gamma-aminobutyric acid (GABA) acting through $\mathrm{GABA}_{\mathrm{B}}$-receptor signaling could eliminate the promigratory effect of norepinephrine, resulting in a reduction in the migration of SW 480 colon carcinoma cells in vitro. Drell et al (109) similarly demonstrated that GABA can block norepinephrine stimulated chemotaxis in the MDA-MB-468 human breast cancer cell line.

\section{Conclusions}

Much knowledge about how cancer cells attain malignant phenotypes, invade, metastasize, and colonize various sites throughout the body has been gained in the last decade. Importantly, deciphering the intricate networks between various genetic lesions and the tumor micro-environment have aided in the understanding of the malignant process, and have suggested mechanisms for tumor cell acquisition of resistance to standard chemotherapeutic interventions. Recurrent themes for promising therapeutic targeting strategies in a variety of malignancies are emerging, however, the complex interactions between cancer cells and their microenvironments, particularly in advanced metastatic disease, makes it unlikely for success to be achieved using an approach directed to only one aspect of cancer progression. Therefore, combination therapies targeting several pathways simultaneously are likely to be most beneficial. Furthermore, methods to selectively target treatments to the cancerous cells by tailoring drug delivery systems to recognize cell surface molecules such as glycans will likely increase the efficacy of both novel and standard therapies with less associated toxicity. Clearly the suppression of metastatic tumor growth would have a major impact on the outcome of many solid tumors and would improve the duration and quality of life for many patients with cancer. The next several years hold much promise for exciting advances in this area of research, which will change the face of cancer therapy in the future.

\section{Acknowledgements}

We would like to thank Vessela Brakalova for helping design Fig. 1. Elitza Germanov would like to thank the Nova Scotia Health Research Foundation for providing studentship funding. 


\section{References}

1. Fidler IJ: The pathogenesis of cancer metastasis: the 'seed and soil' hypothesis revisited. Nat Rev Cancer 3: 453-458, 2003.

2. Sierra A: Metastases and their microenvironments: linking pathogenesis and therapy. Drug Resist Updat 8: 247-257, 2005.

3. Papetti M and Herman IM: Mechanisms of normal and tumorderived angiogenesis. Am J Physiol Cell Physiol 282: 947-970, 2002.

4. Wittekind $\mathrm{C}$ and Neid M: Cancer invasion and metastasis. Oncology 69 (Suppl 1): 14-16, 2005.

5. Fuster MM and Esko JD: The sweet and sour of cancer: glycans as novel therapeutic targets. Nat Rev Cancer 5: 526-542, 2005.

6. Entschladen F, Drell TL IV, Lang K, Joseph J and Zaenker KS Neurotransmitters and chemokines regulate tumor cell migration: potential for a new pharmacological approach to inhibit invasion and metastasis development. Curr Pharm Des 11: 403-411, 2005.

7. Onn A, Tsuboi M and Thatcher N: Treatment of non-small cell lung cancer: a perspective on the recent advances and the experience with gefitinib. Br J Cancer 91 (Suppl 2): S11-S17, 2004.

8. Eccles SA: Targeting key steps in metastatic tumour progression. Curr Opin Genet Dev 15: 77-86, 2005.

9. Traxler P, Bold G, Buchdunger E, et al: Tyrosine kinase inhibitors: from rational design to clinical trials. Med Res Rev 21: 499-512, 2001.

10. Pao W and Miller VA: Epidermal growth factor receptor mutations, small-molecule kinase inhibitors, and non-small cell lung cancer: current knowledge and future directions. J Clin Oncol 23: 2556-2568, 2005.

11. Goldman CK, Kim J, Wong WL, King V, Brock T and Gillespie GY: Epidermal growth factor stimulates vascular endothelial growth factor production by human malignant glioma cells: a model of glioblastoma multiforme pathophysiology. Mol Biol Cell 4: 121-133, 1993.

12. Yamanaka Y, Friess H, Kobrin MS, Buchler M, Beger HG and Korc M: Coexpression of epidermal growth factor receptor and ligands in human pancreatic cancer is associated with enhanced tumor aggressiveness. Anticancer Res 13: 565-569, 1993.

13. Silvestri GA and Rivera MP: Targeted therapy for the treatment of advanced non-small cell lung cancer: a review of the epidermal growth factor receptor antagonists. Chest 128: 3975-3984, 2005.

14. Baker CH, Solorzano CC and Fidler IJ: Blockade of vascular endothelial growth factor receptor and epidermal growth factor receptor signaling for therapy of metastatic human pancreatic cancer. Cancer Res 62: 1996-2003, 2002

15. Kim SJ, Uehara H, Karashima T, Shepherd DL, Killion JJ and Fidler IJ: Blockade of epidermal growth factor receptor signaling in tumor cells and tumor-associated endothelial cells for therapy of androgen-independent human prostate cancer growing in the bone of nude mice. Clin Cancer Res 9: 1200-1210, 2003.

16. Hoekstra R, Dumez H, Eskens FA, et al: Phase I and pharmacologic study of PKI166, an epidermal growth factor receptor tyrosine kinase inhibitor, in patients with advanced solid malignancies. Clin Cancer Res 11: 6908-6915, 2005.

17. Fukuoka M, Yano S, Giaccone G, et al: Multi-institutional randomized phase II trial of gefitinib for previously treated patients with advanced non-small cell lung cancer (The IDEAL 1 Trial) corrected. J Clin Oncol 21: 2237-2246, 2003.

18. Kris MG, Natale RB, Herbst RS, et al: Efficacy of gefitinib, an inhibitor of the epidermal growth factor receptor tyrosine kinase, in symptomatic patients with non-small cell lung cancer: a randomized trial. JAMA 290: 2149-2158, 2003.

19. Lynch TJ, Bell DW, Sordella R, et al: Activating mutations in the epidermal growth factor receptor underlying responsiveness of non-small cell lung cancer to gefitinib. N Engl J Med 350: 2129-2139, 2004.

20. Paez JG, Janne PA, Lee JC, et al: EGFR mutations in lung cancer: correlation with clinical response to gefitinib therapy. Science 304: 1497-1500, 2004.

21. Pao W, Miller V, Zakowski M, et al: EGF receptor gene mutations are common in lung cancers from 'never smokers' and are associated with sensitivity of tumors to gefitinib and erlotinib. Proc Natl Acad Sci USA 101: 13306-13311, 2004.

22. Pao W, Miller VA, Politi KA, et al: Acquired resistance of lung adenocarcinomas to gefitinib or erlotinib is associated with a second mutation in the EGFR kinase domain. PLoS Med 2: e73, 2005.
23. Cappuzzo F, Hirsch FR, Rossi E, et al: Epidermal growth factor receptor gene and protein and gefitinib sensitivity in non-smallcell lung cancer. J Natl Cancer Inst 97: 643-655, 2005.

24. Pao W, Wang TY, Riely GJ, et al: KRAS mutations and primary resistance of lung adenocarcinomas to gefitinib or erlotinib. PLoS Med 2: e17, 2005.

25. Romond EH, Perez EA, Bryant J, et al: Trastuzumab plus adjuvant chemotherapy for operable HER2-positive breast cancer. N Engl J Med 353: 1673-1684, 2005.

26. Safran H, Iannitti D, Ramanathan R, et al: Herceptin and gemcitabine for metastatic pancreatic cancers that overexpress HER-2/neu. Cancer Invest 22: 706-712, 2004.

27. Safran H, Di Petrillo T, Nadeem A, et al: Trastuzumab, paclitaxel, cisplatin, and radiation for adenocarcinoma of the esophagus: a phase I study. Cancer Invest 22: 670-677, 2004.

28. Salesse S and Verfaillie CM: BCR/ABL: from molecular mechanisms of leukemia induction to treatment of chronic myelogenous leukemia. Oncogene 21: 8547-8559, 2002.

29. Branford S, Rudzki Z, Harper A, et al: Imatinib produces significantly superior molecular responses compared to interferon alpha plus cytarabine in patients with newly diagnosed chronic myeloid leukemia in chronic phase. Leukemia 17: 2401-2409, 2003.

30. Chiang KC, Chen TW, Yeh CN, Liu FY, Lee HL and Jan YY: Advanced gastrointestinal stromal tumor patients with complete response after treatment with imatinib mesylate. World J Gastroenterol 12: 2060-2064, 2006.

31. Hampton T: Looking beyond imatinib: next line of targeted drugs for CML shows promise. JAMA 295: 369-370, 2006.

32. Radford IR: Imatinib. Novartis. Curr Opin Investig Drugs 3: 492-499, 2002.

33. Hakomori S: Tumor malignancy defined by aberrant glycosylation and sphingo(glyco)lipid metabolism. Cancer Res 56: 5309-5318, 1996.

34. Iida J, Meijne AM, Knutson JR, Furcht LT and McCarthy JB Cell surface chondroitin sulfate proteoglycans in tumor cell adhesion, motility and invasion. Semin Cancer Biol 7: 155-162, 1996.

35. Bernfield M, Gotte M, Park PW, Reizes O, Fitzgerald ML, Lincecum J and Zako M: Functions of cell surface heparan sulfate proteoglycans. Annu Rev Biochem 68: 729-777, 1999.

36. Wang L, Fuster M, Sriramarao P and Esko JD: Endothelial heparan sulfate deficiency impairs L-selectin- and chemokinemediated neutrophil trafficking during inflammatory responses. Nat Immunol 6: 902-910, 2005.

37. Muller A, Homey B, Soto H, Ge N, Catron D, Buchanan ME, McClanahan T, Murphy E, Yuan W, Wagner SN, Barrera JL, Mohar A, Verastegui E and Zlotnik A: Involvement of chemokine receptors in breast cancer metastasis. Nature 410: 50-56, 2001.

38. Goldshmidt O, Zcharia E, Abramovitch R, et al: Cell surface expression and secretion of heparanase markedly promote tumor angiogenesis and metastasis. Proc Natl Acad Sci USA 99: 10031-10036, 2002.

39. Vlodavsky I, Goldshmidt O, Zcharia E, et al: Molecular properties and involvement of heparanase in cancer progression and normal development. Biochimie 83: 831-839, 2001.

40. Maeder T: Sweet medicines. Sci Am 287: 40-47, 2002.

41. Roy M, Reiland J, Murry BP, Chouljenko V, Kousoulas KG and Marchetti D: Antisense-mediated suppression of Heparanase gene inhibits melanoma cell invasion. Neoplasia 7: 253-262, 2005.

42. Holmberg LA and Sandmaier BM: Vaccination with Theratope (STn-KLH) as treatment for breast cancer. Expert Rev Vaccines 3: 655-663, 2004

43. Holmberg LA, Oparin DV, Gooley T and Sandmaier BM: The role of cancer vaccines following autologous stem cell rescue in breast and ovarian cancer patients: experience with the STnKLH vaccine (Theratope). Clin Breast Cancer 3 (Suppl 4): S144-S151, 2003.

44. de Kleijn EM and Punt CJ: Biological therapy of colorectal cancer. Eur J Cancer 38: 1016-1022, 2002.

45. Ragupathi G, Livingston PO, Hood C, et al: Consistent antibody response against ganglioside GD2 induced in patients with melanoma by a GD2 lactone-keyhole limpet hemocyanin conjugate vaccine plus immunological adjuvant QS-21. Clin Cancer Res 9: 5214-5220, 2003.

46. Kawano T, Cui J, Koezuka Y, et al: Natural killer-like nonspecific tumor cell lysis mediated by specific ligand-activated Valpha14 NKT cells. Proc Natl Acad Sci USA 95: 5690-5693, 1998. 
47. Smyth MJ, Crowe NY, Hayakawa Y, Takeda K, Yagita H and Godfrey DI: NKT cells - conductors of tumor immunity? Curr Opin Immunol 14: 165-171, 2002.

48. Smyth MJ, Crowe NY, Pellicci DG, et al: Sequential production of interferon-gamma by NK1.1(+) T cells and natural killer cells is essential for the antimetastatic effect of alpha-galactosylceramide. Blood 99: 1259-1266, 2002.

49. Toura I, Kawano T, Akutsu Y, Nakayama T, Ochiai T and Taniguchi M: Cutting edge: inhibition of experimental tumor metastasis by dendritic cells pulsed with alpha-galactosylceramide. J Immunol 163: 2387-2391, 1999.

50. Akutsu Y, Nakayama T, Harada M, et al: Expansion of lung V alpha 14 NKT cells by administration of alpha-galactosylceramide-pulsed dendritic cells. Jpn J Cancer Res 93: 397-403, 2002.

51. Ishikawa A, Motohashi S, Ishikawa E, et al: A phase I study of alpha-galactosylceramide (KRN7000)-pulsed dendritic cells in patients with advanced and recurrent non-small cell lung cancer. Clin Cancer Res 11: 1910-1917, 2005.

52. Lee CM, Tanaka T, Murai T, et al: Novel chondroitin sulfatebinding cationic liposomes loaded with cisplatin efficiently suppress the local growth and liver metastasis of tumor cells in vivo. Cancer Res 62: 4282-4288, 2002.

53. Bergers $\mathrm{G}$ and Benjamin LE: Tumorigenesis and the angiogenic switch. Nat Rev Cancer 3: 401-410, 2003.

54. Hlatky L, Hahnfeldt P and Folkman J: Clinical application of antiangiogenic therapy: microvessel density, what it does and doesn't tell us. J Natl Cancer Inst 94: 883-893, 2002.

55. Kerbel R and Folkman J: Clinical translation of angiogenesis inhibitors. Nat Rev Cancer 2: 727-739, 2002.

56. Ferrara N, Gerber HP and LeCouter J: The biology of VEGF and its receptors. Nat Med 9: 669-676, 2003.

57. Gerber HP, Malik AK, Solar GP, et al: VEGF regulates haematopoietic stem cell survival by an internal autocrine loop mechanism. Nature 417: 954-958, 2002.

58. Bikfalvi A: Recent developments in the inhibition of angiogenesis: examples from studies on platelet factor- 4 and the VEGF/VEGFR system. Biochem Pharmacol 68: 1017-1021, 2004.

59. Stern M and Herrmann R: Overview of monoclonal antibodies in cancer therapy: present and promise. Crit Rev Oncol Hematol 54: 11-29, 2005.

60. Hurwitz H, Fehrenbacher L, Novotny W, et al: Bevacizumab plus irinotecan, fluorouracil, and leucovorin for metastatic colorectal cancer. N Engl J Med 350: 2335-2342, 2004.

61. Yang JC, Haworth L, Sherry RM, et al: A randomized trial of bevacizumab, an anti-vascular endothelial growth factor antibody, for metastatic renal cancer. N Engl J Med 349: 427-434, 2003.

62. Patel PH, Chaganti RS and Motzer RJ: Targeted therapy for metastatic renal cell carcinoma. Br J Cancer 94: 614-619, 2006.

63. Hainsworth JD, Sosman JA, Spigel DR, Edwards DL, Baughman C and Greco A: Treatment of metastatic renal cell carcinoma with a combination of bevacizumab and erlotinib. J Clin Oncol 23: 7889-7896, 2005.

64. Herbst RS, Johnson DH, Mininberg E, et al: Phase I/II trial evaluating the anti-vascular endothelial growth factor monoclonal antibody bevacizumab in combination with the HER-1/epidermal growth factor receptor tyrosine kinase inhibitor erlotinib for patients with recurrent non-small cell lung cancer. J Clin Oncol 23: 2544-2555, 2005.

65. Johnson DH, Fehrenbacher L, Novotny WF, et al: Randomized phase II trial comparing bevacizumab plus carboplatin and paclitaxel with carboplatin and paclitaxel alone in previously untreated locally advanced or metastatic non-small cell lung cancer. J Clin Oncol 22: 2184-2191, 2004.

66. Wood JM, Bold G, Buchdunger E, et al: PTK787/ZK 222584, a novel and potent inhibitor of vascular endothelial growth factor receptor tyrosine kinases, impairs vascular endothelial growth factor-induced responses and tumor growth after oral administration. Cancer Res 60: 2178-2189, 2000.

67. Subbaramaiah K and Dannenberg AJ: Cyclooxygenase 2: a molecular target for cancer prevention and treatment. Trends Pharmacol Sci 24: 96-102, 2003.

68. Sheng H, Shao J, Washington MK and DuBois RN: Prostaglandin E2 increases growth and motility of colorectal carcinoma cells. J Biol Chem 276: 18075-18081, 2001.

69. Ben-Av P, Crofford LJ, Wilder RL and Hla T: Induction of vascular endothelial growth factor expression in synovial fibroblasts by prostaglandin $\mathrm{E}$ and interleukin-1: a potential mechanism for inflammatory angiogenesis. FEBS Lett 372: 83-87, 1995.
70. Kakiuchi Y, Tsuji S, Tsujii M, et al: Cyclooxygenase-2 activity altered the cell-surface carbohydrate antigens on colon cancer cells and enhanced liver metastasis. Cancer Res 62: 1567-1572, 2002.

71. Kuwano T, Nakao S, Yamamoto H, Tsuneyoshi M, Yamamoto T, Kuwano $\mathrm{M}$ and Ono M: Cyclooxygenase 2 is a key enzyme for inflammatory cytokine-induced angiogenesis. FASEB J 18: 300-310, 2004.

72. Tsuji S, Tsujii M, Kawano S and Hori M: Cyclooxygenase-2 upregulation as a perigenetic change in carcinogenesis. J Exp Clin Cancer Res 20: 117-129, 2001.

73. Sano H, Kawahito Y, Wilder RL, et al: Expression of cyclooxygenase- 1 and -2 in human colorectal cancer. Cancer Res 55 : 3785-3789, 1995.

74. Murata H, Kawano S, Tsuji S, et al: Cyclooxygenase-2 overexpression enhances lymphatic invasion and metastasis in human gastric carcinoma. Am J Gastroenterol 94: 451-455, 1999.

75. Iniguez MA, Rodriguez A, Volpert OV, Fresno M and Redondo JM: Cyclooxygenase-2: a therapeutic target in angiogenesis. Trends Mol Med 9: 73-78, 2003.

76. Steinbach G, Lynch PM, Phillips RK, et al: The effect of celecoxib, a cyclooxygenase-2 inhibitor, in familial adenomatous polyposis. N Engl J Med 342: 1946-1952, 2000.

77. Altorki NK, Keresztes RS, Port JL, et al: Celecoxib, a selective cyclo-oxygenase-2 inhibitor, enhances the response to preoperative paclitaxel and carboplatin in early-stage non-small cell lung cancer. J Clin Oncol 21: 2645-2650, 2003.

78. Bellon G, Martiny L and Robinet A: Matrix metalloproteinases and matrikines in angiogenesis. Crit Rev Oncol Hematol 49: 203-220, 2004.

79. Cao Y, Chen A, An SS, Ji RW, Davidson D and Llinas M: Kringle 5 of plasminogen is a novel inhibitor of endothelial cell growth. J Biol Chem 272: 22924-22928, 1997.

80. Lucas R, Holmgren L, Garcia I, et al: Multiple forms of angiostatin induce apoptosis in endothelial cells. Blood 92: 4730-4741, 1998.

81. O'Reilly MS, Holmgren L, Shing Y, et al: Angiostatin: a novel angiogenesis inhibitor that mediates the suppression of metastases by a Lewis lung carcinoma. Cell 79: 315-328, 1994.

82. Cao Y, O'Reilly MS, Marshall B, Flynn E, Ji RW and Folkman J: Expression of angiostatin cDNA in a murine fibrosarcoma suppresses primary tumor growth and produces long-term dormancy of metastases. J Clin Invest 101: 1055-1063, 1998.

83. Sonveaux P, Brouet A, Havaux X, Gregoire V, Dessy C, Balligand JL and Feron O: Irradiation-induced angiogenesis through the up-regulation of the nitric oxide pathway: implications for tumor radiotherapy. Cancer Res 63: 1012-1019, 2003.

84. Mauceri HJ, Hanna NN, Beckett MA, et al: Combined effects of angiostatin and ionizing radiation in antitumour therapy. Nature 394: 287-291, 1998

85. Rao JS, Gondi C, Chetty C, Chittivelu S, Joseph PA and Lakka SS: Inhibition of invasion, angiogenesis, tumor growth, and metastasis by adenovirus-mediated transfer of antisense uPAR and MMP-9 in non-small cell lung cancer cells. Mol Cancer Ther 4: 1399-1408, 2005.

86. Sparano JA, Bernardo P, Stephenson P, Gradishar WJ, Ingle JN, Zucker S and Davidson NE: Randomized phase III trial of marimastat versus placebo in patients with metastatic breast cancer who have responding or stable disease after first-line chemotherapy: Eastern Cooperative Oncology Group trial E2196. J Clin Oncol 22: 4683-4690, 2004.

87. Bissett D, O'Byrne KJ, von Pawel J, et al: Phase III study of matrix metalloproteinase inhibitor prinomastat in non-small cell lung cancer. J Clin Oncol 23: 842-849, 2005.

88. Nozaki S, Endo Y, Nakahara H, et al: Inhibition of invasion and metastasis in oral cancer by targeting urokinase-type plasminogen activator receptor. Oral Oncol 41: 971-977, 2005.

89. Martinez-Zaguilan R, Seftor EA, Seftor RE, Chu YW, Gillies RJ and Hendrix MJ: Acidic $\mathrm{pH}$ enhances the invasive behavior of human melanoma cells. Clin Exp Metastasis 14: 176-186, 1996.

90. Sennoune SR, Bakunts K, Martinez GM, Chua-Tuan JL, Kebir Y, Attaya MN and Martinez-Zaguilan R: Vacuolar $\mathrm{H}^{+}$-ATPase in human breast cancer cells with distinct metastatic potential: distribution and functional activity. Am J Physiol Cell Physiol 286: C1443-C1452, 2004.

91. Lu X, Qin W, Li J, Tan N, et al: The growth and metastasis of human hepatocellular carcinoma xenografts are inhibited by small interfering RNA targeting to the subunit ATP6L of proton pump. Cancer Res 65: 6843-6849, 2005. 
92. Xu L and Fidler IJ: Interleukin 8: an autocrine growth factor for human ovarian cancer. Oncol Res 12: 97-106, 2000.

93. Fukumura D, Xu L, Chen Y, Gohongi T, Seed B and Jain RK: Hypoxia and acidosis independently up-regulate vascular endothelial growth factor transcription in brain tumors in vivo. Cancer Res 61: 6020-6024, 2001

94. Itoh T, Tanioka M, Yoshida H, Yoshioka T, Nishimoto H and Itohara S: Reduced angiogenesis and tumor progression in gelatinase A-deficient mice. Cancer Res 58: 1048-1051, 1998.

95. Balkwill F: Cancer and the chemokine network. Nat Rev Cancer 4: 540-550, 2004

96. Tanaka T, Bai Z, Srinoulprasert Y, Yang BG, Hayasaka H and Miyasaka M: Chemokines in tumor progression and metastasis. Cancer Sci 96: 317-322, 2005.

97. Hanahan D and Weinberg RA: The hallmarks of cancer. Cell 100: $57-70,2000$

98. Balkwill F: The significance of cancer cell expression of the chemokine receptor CXCR4. Semin Cancer Biol 14: 171-179, 2004.

99. Ganju RK, Brubaker SA, Meyer J, Dutt P, Yang Y, Qin S, Newman W and Groopman JE: The alpha-chemokine, stromal cell-derived factor-1alpha, binds to the transmembrane Gprotein-coupled CXCR-4 receptor and activates multiple signal transduction pathways. J Biol Chem 273: 23169-23175, 1998.

100.Guleng B, Tateishi K, Ohta M, et al: Blockade of the stromal cell-derived factor-1/CXCR4 axis attenuates in vivo tumor growth by inhibiting angiogenesis in a vascular endothelial growth factor-independent manner. Cancer Res 65: 5864-5871, 2005.

101. Rubin JB, Kung AL, Klein RS, et al: A small-molecule antagonist of CXCR4 inhibits intracranial growth of primary brain tumors. Proc Natl Acad Sci USA 100: 13513-13518, 2003.

102. Wiley HE, Gonzalez EB, Maki W, Wu MT and Hwang ST: Expression of CC chemokine receptor-7 and regional lymph node metastasis of B16 murine melanoma. J Natl Cancer Inst 93: $1638-1643,2001$.
103. Mashino K, Sadanaga N, Yamaguchi H, Tanaka F, Ohta M, Shibuta K, Inoue $\mathrm{H}$ and Mori M: Expression of chemokine receptor CCR7 is associated with lymph node metastasis of gastric carcinoma. Cancer Res 62: 2937-2941, 2002.

104. Ding Y, Shimada Y, Maeda M, Kawabe A, Kaganoi J, Komoto I, Hashimoto Y, Miyake M, Hashida $\mathrm{H}$ and Imamura M: Association of CC chemokine receptor 7 with lymph node metastasis of esophageal squamous cell carcinoma. Clin Cancer Res 9: 3406-3412, 2003

105. Tilly BC, Tertoolen LG, Remorie R, Ladoux A, Verlaan I, de Laat SW and Moolenaar WH: Histamine as a growth factor and chemoattractant for human carcinoma and melanoma cells: action through $\mathrm{Ca} 2(+)$-mobilizing $\mathrm{H} 1$ receptors. J Cell Biol 110: 1211-1215, 1990.

106. Baba K and Yamaguchi O: Effects of bradykinin on cytoplasmic calcium and motility in murine bladder tumor cells. J Urol 165: 259-262, 2001

107. Andersson OK, Almgren T, Persson B, Samuelsson O, Hedner T and Wilhelmsen L: Survival in treated hypertension: follow up study after two decades. BMJ 317: 167-171, 1998.

108. Joseph J, Niggemann B, Zaenker KS and Entschladen F: The neurotransmitter gamma-aminobutyric acid is an inhibitory regulator for the migration of SW 480 colon carcinoma cells. Cancer Res 62: 6467-6469, 2002

109.Drell TL IV, Joseph J, Lang K, Niggemann B, Zaenker KS and Entschladen F: Effects of neurotransmitters on the chemokinesis and chemotaxis of MDA-MB-468 human breast carcinoma cells. Breast Cancer Res Treat 80: 63-70, 2003.

110. Kakkar AK: An expanding role for antithrombotic therapy in cancer patients. Cancer Treat Rev 29 (Suppl 2): 23-26, 2003.

111. Hayakawa Y, Rovero S, Forni G and Smyth MJ: Alphagalactosylceramide (KRN7000) suppression of chemical- and oncogene-dependent carcinogenesis. Proc Natl Acad Sci USA 100: 9464-9469, 2003. 